\title{
Tailoring ECC for Special Attributes: A Review
}

\author{
Victor C. Li*
}

(Received July 9, 2012, Accepted August 3, 2012, Published online September 18, 2012)

\begin{abstract}
This article reviews the tailoring of engineered cementitious composites (ECC), a type of high performance fiber reinforced cementitious composites with a theoretical design basis, for special attributes or functions. The design basis, a set of analytic tools built on micromechanics, provides guidelines for tailoring of fiber, matrix, and fiber/matrix interfaces to attain tensile ductility in ECC. If conditions for controlled multiple cracking are disturbed by the need to introduce ingredients to attain a special attribute or function, micromechanics then serve as a systematic and rational means to efficiently recover composite tensile ductility. Three examples of ECCs with attributes of lightweight, high early strength, and self-healing functions, are used to illustrate these tailoring concepts. The fundamental approach, however, is broadly applicable to a wide variety of ECCs designed for targeted fresh and/or hardened characteristics required for specific applications.
\end{abstract}

Keywords: engineered cementitious composites, HPFRCC, tailoring, functions, micromechanics.

\section{Introduction}

Concrete is not a single material. There are various types of concrete, including high strength concrete, lightweight concrete, and self-consolidating concrete that are designed to have fresh or hardened properties that match the requirements of a specific application. Even within a given structure, a building for example, concrete with different strength class may be used. The idea of designing a mix that leads to a desired construction or structural performance has been fully accepted in the concrete industry for many years.

The mechanical property of concrete that is designed or controlled is typically its compressive strength, since this is the most important material characteristic in concrete specification and in building codes. In recent years, however, other characteristics of concrete, such as durability, are coming to the forefront. It is generally recognized that infrastructure durability is often associated with concrete cracks resulting from concrete brittleness. High compressive strength or densely packed concrete does not overcome brittleness. If nothing else, high compressive strength can be more brittle; some high strength concrete infrastructures have indicated a higher tendency for early age cracking. Most durability studies in the laboratory are conducted with specimens that are not loaded, such as permeability measurement. This has given credence to high strength or high dense concrete as being durable, but its field performance

Department of Civil and Environmental Engineering, University of Michigan, Ann Arbor, MI 48109-2125, USA. *Corresponding Author; E-mail: vcli@umich.edu Copyright ( $\odot$ The Author(s) 2012. This article is published with open access at Springerlink.com under restrained condition and under loading has not always reflected this anticipation.

While the importance of controlling brittleness and crack width has been gaining attention with regard to infrastructure durability, the design of such concrete has not been given adequate attention. The tools for designing concrete with controlled cracking and crack width are limited, and for designing concrete with crack control together with other useful attributes such as low density, high early strength, and controlled thermal response are all but absent.

In recent years, engineered cementitious composites, or ECC for short, has been developed to address the brittleness shortcomings of normal concrete. ECC has tensile ductility as much as three hundred times that of normal concrete, so that the shape of its tensile stress-strain curve looks more like that of steel than normal concrete. For this reason, it is also referred to as strainhardening cementitious composites (or SHCC).

Figure 1 shows the stress-strain relationship of a most studied version of ECC-ECC M45 (Wang 2005). Apart from the high tensile ductility of over $3 \%$, this figure also shows the self-controlled averaged tight crack width development of about $60 \mu \mathrm{m}$ even when strained to beyond $1 \%$. Normal concrete fractures at about $0.01 \%$ with a localized crack of indefinite width.

Engineered cementitious composites is a family of engineered high performance fiber reinforced cementitious composites. While the fundamental characteristic of ECC is its extreme tensile ductility, a variety of useful attributes/ functions have recently been designed into particular members of this material family. These special attributes/functions include light-weightedness, low carbon footprint, selfhealing, self-sensing, self-thermal control, impact resistant, fire resistive with low thermal conductivity, pigmentability, rapid setting, self-consolidating, sprayability, extrudability, 


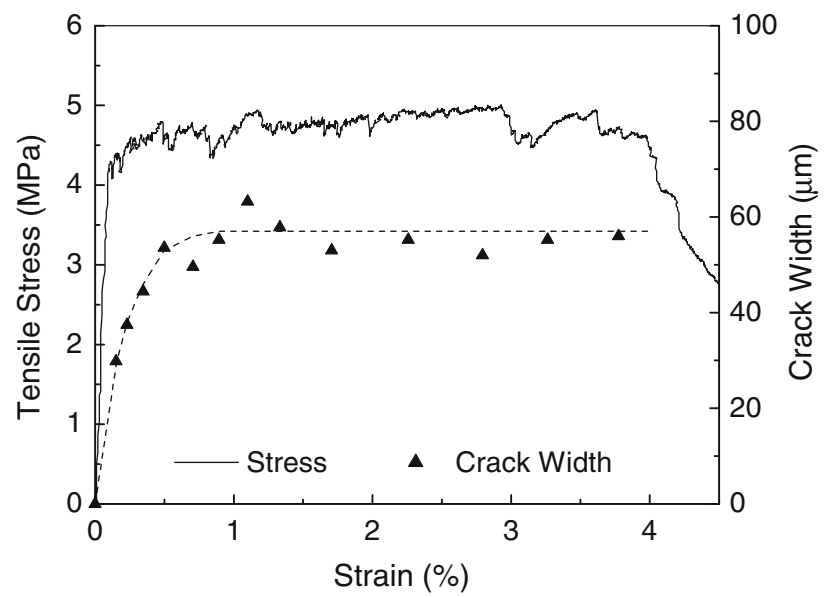

Fig. 1 Stress-strain curve and averaged crack width development of ECC M45.

and others. Often, the incorporation of these special attributes/functions requires the modification of the composition that may negatively limit or even eliminate the fundamental tensile ductility characteristic of ECC. Fortunately, past experience has demonstrated that there is enough design flexibility so that appropriate composition readjustments can allow the tensile ductility to be restored. The key to systematic composition modification and readjustment to attain special attributes/functions while maintaining the fundamental ductile characteristic of ECC is the micromechanics based analytic tool set.

In this article, we review the essence of the micromechanics based analytic tool set, emphasizing its use in controlling the composite matrix, fiber, and fiber/matrix interface ingredients for achieving targeted properties and special attributes. For illustration of this approach, we shall discuss three example ECC materials with the attributes/ functions of light-weightedness, high early strength, and self-healing, respectively. These example materials were chosen to highlight the effects of controlling the matrix fracture toughness, the fiber/matrix interface, and the matrix flaw size on the composite behavior. In each case, ECC M45 was used as a template, with ingredient modifications made to attain the special attributes, and then adjusted as necessary to maintain the unique tensile ductility of ECCs. It is emphasized that the availability of the micromechanics based analytic tool set removes a lot of guesswork, which allows systematic and effective tailoring of material ingredients to efficiently attain the desired material behavior.

\section{Micromechanics Based Analytic Tool Set}

ECC derives its tensile ductility by deliberately allowing cracks to grow out from pre-existing flaws in a controlled manner. The pre-existing flaws may be a result of naturally entrapped air bubbles or may be deliberately implanted as artificial flaws. Unlike the design of ultra high strength concrete, ECC benefits from the pre-existing flaws by managing their size range rather than by eliminating them.
Typically, the damage pattern under uniaxial tension is a large number of closely spaced microcracks of width less than $100 \mu \mathrm{m}$, spaced a few millimeters apart. In this manner, localized catastrophic brittle fracture is suppressed. The ductility of ECC is the sum total of distributed deformation resulting from the diffused microcrack damage.

To attain the multiple microcracking response, two complimentary criteria have been proposed - these are the Strength criterion and the Energy criterion, and they are summarized below. Details of the micromechanical model can be found in Li et al. (2001).

\subsection{Strength Criterion}

The strength criterion states that the tensile stress $\sigma_{\mathrm{cr}}$ to initiate a crack from a pre-existing flaw must be below the bridging capacity of the fibers $\sigma_{0}$ crossing that crack. That is,

$$
\sigma_{c r}<\sigma_{o}
$$

Satisfaction of this strength criterion assures that the initiated crack will not result in catastrophic loss of load carrying capacity on this crack plane. The matrix cracking strength $\sigma_{c r}$ is dependent on the initial flaw size $c$ and the matrix fracture toughness $K_{m}$. In most cases, the matrix fracture toughness can be assumed to be uniform throughout the material, but flaw sizes do vary in space. As a result, crack initiation starts at the largest and most favorably oriented flaw and progressively works its way to smaller and smaller flaws as the tensile load increases. In other words, the microcrack density typically increases with increasing tensile load. Crack spacing decreases with each additional microcrack formed.

On each crack plane, the load shed by the matrix is taken over by the bridging fibers. The load carried by the bridging fiber is a function of the opening of the crack, characterized by a $\sigma(\delta)$ relationship that increases to a peak and then decreases. The peak value $\sigma_{0}$, termed as the fiber bridging capacity, varies from one crack plane to another due to the inevitable spatial non-uniformity in fiber dispersion. If Eq. (1) is violated on any given crack plane, that crack localizes into a fracture that terminates the multiple cracking process. If this occurs on the very first crack, only one crack will be present followed by a tension-softening process. This is the typical case for ordinary fiber reinforced concrete. Very small flaws with $\sigma_{c r}$ higher than the lowest $\sigma_{0}$ on already existing crack planes will never be activated.

Clearly, to maintain (1) in the face of material variability, a high margin or difference between $\sigma_{0}$ and $\sigma_{c r}$ is preferred. In order words, a high $\left(\sigma_{0} / \sigma_{c r}\right)$ ratio is desired (Kanda and $\mathrm{Li}$ 2006). Low $\sigma_{c r}$ can be attained by lowering the matrix fracture toughness $K_{m}$ or by having large flaw size $c$. However, excessive lowering of $K_{m}$ or large flaw size $c$ could lead to low first crack strength and compressive strength. A more preferred scenario is to enhance $\sigma_{0}$. This can be achieved with a high fiber volume fraction $V_{f}$, a strong fiber, and/or strong fiber/matrix bond. A high fiber volume fraction, however, implies high cost, poor workability, and inhomogeneous fiber dispersion. A strong fiber typically incurs 
higher material cost. A strong fiber/matrix bond can be engineered; however, excessively high bond leads to fiber rupture that violates the energy criterion, as explained below. Maintaining the strength criterion for multiple cracking while satisfying first cracking and compressive strength, cost, and workability requirements represents a delicate balance when designing ECC ( $\mathrm{Li}$ et al. 2001, 2002).

\subsection{Energy Criterion}

After a crack initiates from a pre-existing flaw, the manner in which this crack propagates dictates whether the bridging fibers will be ruptured or pulled out. A lot of this depends on the opening magnitude of the crack. If crack opening is large, rupturing or pulling out of fibers on the crack flanks will be inevitable as the crack propagates. In an ideally brittle material, the crack opening is known to scale parabolically with distance behind the crack tips, which means that the crack opening will increase indefinitely with the propagating crack length. In normal fiber reinforced concrete, the parabolic shaped opening near the crack tip is modified by the closing pressure of bridging fibers, but otherwise follows the same trend in terms of crack opening scaling with the crack length. This implies that the bridged crack must undergo tension softening as fibers are ruptured or pulled out in such composites. This is the normal scenario for ordinary fiber reinforced concrete.

In ECC, fiber rupture or pullout is carefully controlled by restricting the opening of the propagating cracks, typically to below $100 \mu \mathrm{m}$. To achieve this, a different mode of crack propagation is required. This is the steady state flat crack propagation mode in which crack opening is uniform at $\delta_{s s}$ except for a small region behind the crack tip. This flat crack propagation mode requires a balance of energy so that the work done due to applied tensile load $\sigma_{s s}$ on the body must equate to the energy required to break down the crack tip material $J_{t i p}$ and the energy required to open the crack against fiber bridging from 0 to $\delta_{s s}$. This concept can be summarized as

$$
\sigma_{s s} \delta_{s s}-\int_{0}^{\delta_{s s}} \sigma(\delta) d \delta=J_{t i p}
$$

where

$$
J_{\text {tip }}=\frac{K_{m}^{2}}{E_{c}}
$$

and $E_{c}$ is the composite Young's Modulus. The left hand side of (2) represents the area to the left of the $\sigma(\delta)$ relation at $\sigma_{\mathrm{ss}}\left(\delta_{\mathrm{ss}}\right)$, often abbreviated as the complementary energy. Since the $\sigma(\delta)$ relation goes through a peak $\sigma_{0}\left(\delta_{\mathrm{o}}\right)$, the complementary energy has the maximum value of

$$
J_{b}^{\prime} \equiv \sigma_{o} \delta_{o}-\int_{0}^{\delta_{o}} \sigma(\delta) d \delta
$$

To ensure flat crack propagation mode, therefore, $J_{b}^{\prime}$ must exceed $J_{\text {tip }}$, or

$$
J_{b}^{\prime}>J_{t i p}
$$

Equation (5) represents the energy criterion for multiple cracking. A high ratio of $J_{b}{ }_{b} / J_{\text {tip }}$ is preferred for robust multiple cracking in the presence of material variability (Kanda and Li 2006).

$J_{t i p}$ is the energy equivalent of the matrix fracture toughness $K_{m} . J^{\prime}{ }_{b}$ is directly related to the fiber and fiber/matrix interface properties. Detailed expressions for $\sigma(\delta)$ relationships have been previously derived for fibers and interfaces with various characteristics (Lin and $\mathrm{Li}$ 1997). Most importantly, Eq. (4) indicates that excessively low or high interfacial bond is not preferred since it suppresses the $J^{\prime}{ }_{b}$ value. In other words, the selection of fiber and the control of interfacial parameters are paramount to robust multiple cracking and tensile ductility in ECC.

The dual criteria for multiple cracking provide systematic guidance for designing ECC. Specifically, a low matrix fracture toughness $K_{m}$ (or $J_{t i p}$ ), appropriate flaw size $c$, and a high complimentary energy $J_{b}^{\prime}$ are conducive to multiple cracking.

$K_{m}$ and $c$ are properties of the matrix material. They are influenced by the binder and aggregate types and size, $w / c$ ratio, and other factors. Typically, a more reactive binder (e.g. cement vs mineral admixtures) would lead to a higher $K_{m}$, as would a given binder with finer particle size (more reactive surfaces). Larger aggregate size tends to increase $K_{m}$ due to the creation of a more tortuous crack path. For both binder and aggregates, the consideration is to limit the energy barrier to microcrack initiation and propagation. The initial flaw size distribution is also a function of matrix material design. Typically, flaws of a few mm exist due to trapped air during mixing, and may be influenced by workability (especially when fibers are included). Some chemical admixtures also generate air bubbles. However, if the correct range of natural flaw sizes is not present, artificial means to introduce them can be considered. In other words, flaws in ECC are to be managed, not eliminated. For matrix composition in ECC, therefore, the binder, aggregate, and flaw size population are the most important design parameters.

The (maximum) complimentary energy $J_{b}{ }_{b}$ is a property of the fiber and fiber/matrix interface. High fiber strength is necessary to maintain a high $\sigma_{0}$ without fiber rupture. High fiber stiffness is helpful to maintain tight crack width but may not be conducive to high $J^{\prime}{ }_{b}$. High fiber aspect (length to diameter) ratio is preferred; although this is limited by workability as mixing with high aspect ratio fibers tends to create fiber balling and inhomogeneous dispersion. Excessively high aspect ratio also may lead to excessive amount of fiber rupture. Perhaps the most important factors controlling $J_{b}^{\prime}$ are the fiber/matrix interfacial properties - specifically the chemical and frictional bonds. Contrary to intuition, high chemical bond which limits debonding of fiber from matrix is not preferred as it suppresses the $J^{\prime}{ }_{b}$ value. Instead, a high frictional bond during fiber slippage is advantageous to maintaining adequate load carrying capacity across the 
multiple cracks while allowing crack opening to occur in a controlled (non-catastrophic) manner. In ECC, the interface is designed to have a relatively low chemical bond, and the frictional bond actually increases with slippage, i.e. sliphardening. This can be accomplished by an appropriate amount of wettability of the fiber surface in combination with appropriate densification in the interfacial transition zone ( $\mathrm{Li}$ et al. 2002). In the case of excessively hydrophilic surfaces, a thin coating could be used to introduce wettability. In the case of fibers with hydrophobic surfaces, it may be necessary to enhance bonding of the fiber to cement via polar group implantation using techniques such as plasma treatment (Wu and $\mathrm{Li}$ 1999). Tuning the fiber's mechanical and geometric properties and the fiber/matrix interface properties for a given matrix is a key element in ECC design.

From the above discussions, it is clear that choosing (or designing) the right fiber is important in attaining multiple cracking and tensile ductility in ECC. What is often overlooked is the equally important matrix and fiber/matrix interface controls. Having a high performance fiber combined with a poorly matching matrix and/or interface will lead to an ordinary fiber reinforced concrete with single crack tension-softening behavior.

\section{Tailoring ECC}

As mentioned earlier, ECC can be tailored to exhibit not only ultra-high ductility but also embody special attributes or functions. In this section, we review three ECCs so designed, emphasizing the use of micromechanics based guidance as discussed in the section above.

\subsection{Lightweight ECC}

Lightweight concrete has been designed using a variety of low-density ingredients including shale, entrained air, polymeric beads, and hollow glass bubbles. While achieving light-weightedness, the resulting concrete often becomes even more brittle, with reduced tensile and compressive strengths. Lightweight ECC has been designed to remain ductile and with limited loss of strength. These considerations constrain the type, size, and amount of low-density particles that could be used in lightweight ECC.

To maintain tensile ductility in lightweight ECC, considerations should be given to the effects of lightweight particles on the fiber, matrix, and fiber/matrix interface. Lightweight particles are expected to reduce the density of the interfacial transition zone that already has lower density (higher porosity) than the bulk matrix. This effectively leads to a lower fiber/matrix interfacial bond and suggests the preference of a fiber that has hydrophilic surface. PVA fibers having strong hydrophilic surface with a pendant $-\mathrm{OH}$ group lend itself to this tendency (Horikoshi et al. 2006). The amount of surface coating normally adopted to reduce its excessive hydrophilicity should be appropriately adjusted to account for the impact of lightweight particle inclusion.

The most direct impact of lightweight particles is the lowering of ECC matrix fracture toughness $K_{m}$. For ECC design, this is actually favorable, as discussed in the previous section. The lowering of $K_{m}$ makes the satisfaction of the strength criterion (1) more readily via the reduction in $\sigma_{c r}$. Further, the corresponding reduction in $J_{\text {tip }}$ also enables the satisfaction of the energy criterion (5) more readily. Indeed $J^{\prime}{ }_{b}$ is enhanced due to a reduction in chemical bond, provided the frictional bond does not suffer from an excessive loss. Thus an enlargement of $J^{\prime}{ }_{b} / J_{\text {tip }}$ is feasible with the introduction of appropriate type, size, and amount of lightweight particles.

Wang and Li (2003) investigated lightweight ECCs using four types of lightweight fillers, including air bubbles generated by air entrainment admixture, expanded perlite sand, hollow glass bubbles (two sizes), and polymeric microform. The most successful lightweight ECC was developed with hollow glass bubbles.

Figure 2 shows the tensile stress-strain curve of a lightweight ECC containing hollow glass bubbles of diameter $10-60 \mu \mathrm{m}$ with a mean value of $30 \mu \mathrm{m}$ and density of $0.6 \mathrm{~g} / \mathrm{cm}^{3}$. At a weight ratio of glass bubbles to cement of $20 \%$, its introduction leads to a composite density of $1.45 \mathrm{~g} / \mathrm{cm}^{3}$, tensile ultimate strength and strain of $4.31 \mathrm{MPa}$ and $4.24 \%$ respectively, and a compressive strength of 41.7 MPa. The stress-strain curves of this composite are robust. Figure 3 shows the saturated crack pattern after straining to $4 \%$. The crack width under load is less than $80 \mu \mathrm{m}$. Figure 4 shows the uniform dispersion of PVA fibers

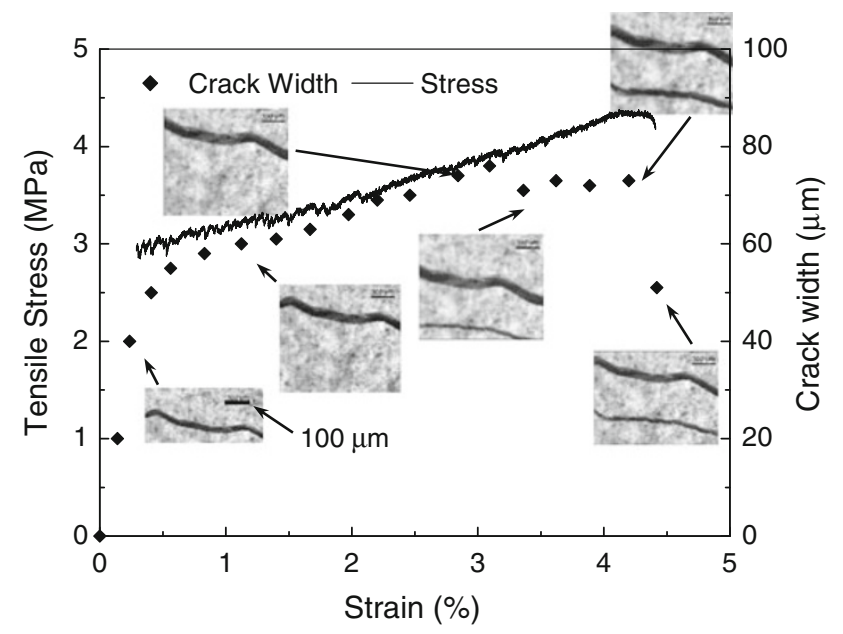

Fig. 2 Tensile stress and crack width vs. strain relationship of a lightweight ECC with density about $60 \%$ that of normal concrete.

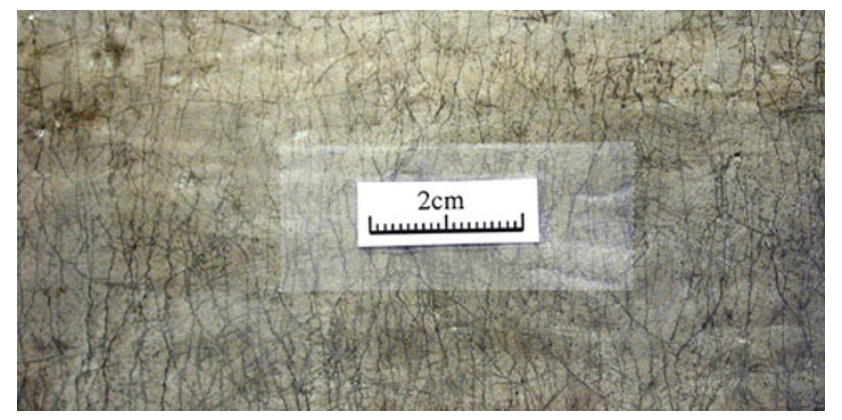

Fig. 3 Saturated crack pattern of lightweight ECC after straining to $4 \%$ in tension. 


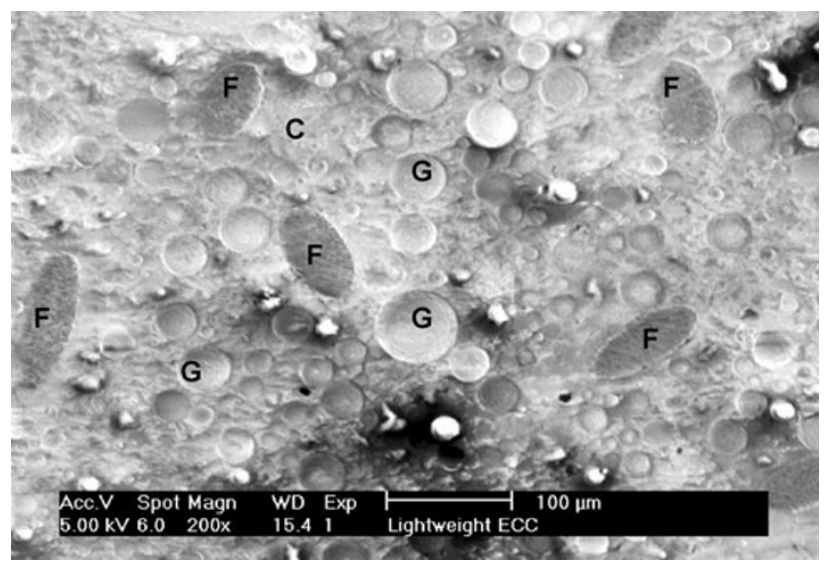

Fig. 4 SEM of a lightweight ECC showing uniformly distributed PVA fibers $(F)$ and a fine cellular structure constructed by glass bubbles $(G)$ and cement hydrates $(C)$.

(F) and a fine cellular structure of glass bubbles (G) and cement hydrates (C). As expected, the fiber/matrix interface bond properties were reduced, although this might be caused by the increase in $w / c$ ratio to maintain workability. The most dramatic change caused by the presence of the hollow glass bubbles in micromechanical parameters was reported to be the matrix fracture toughness, $K_{m}$ (at 28 days) which dropped from $0.64 \pm 0.05 \mathrm{MPa} \sqrt{ } \mathrm{m}$ for ECC M45 to $0.13 \pm 0.01 \mathrm{MPa} \sqrt{ } \mathrm{m}$ for lightweight ECC. This lowering of $K_{m}$ is highly favorable to multiple cracking and tensile ductility of lightweight ECC.

Further reduction in composite density is possible with increase of glass bubble size and content. For example a lightweight ECC mix containing a slightly larger size glass bubble (density $0.38 \mathrm{~g} / \mathrm{cm}^{3}$; size distribution $10-80 \mu \mathrm{m}$ with mean value of $40 \mu \mathrm{m}$ ) at a weight ratio to cement of $50 \%$ drove the ECC composite density down to $0.93 \mathrm{~g} / \mathrm{cm}^{3}$, below the density of water. Remarkably, the tensile ultimate strength and strain capacity was maintained at $2.85 \mathrm{MPa}$ and $3.70 \%$ respectively, although the compressive strength dropped to $21.8 \mathrm{MPa}$. With this compressive strength, however, this ultra lightweight and ductile ECC still qualifies as a structural concrete, while its density is only about $40 \%$ of that of normal concrete.

\subsection{High Early Strength ECC}

The target minimum compressive strength of high early strength ECC (HES-ECC) was set at $17.2 \mathrm{MPa}$ at $4 \mathrm{~h}$ and 48.3 $\mathrm{MPa}$ at 28 days ( $\mathrm{Li}$ and $\mathrm{Li} 2011$ ). These target values were based on a survey of various DOT requirements and FHWA recommendation for high early strength concrete for repairs of bridge or structures that need to be reopened to traffic within 4-6 h after placement.

Several binder modifications were made to ECC M45 to attain the high early age compressive strength. These include the replacement of Type I by Type III Portland cement, the elimination of fly ash, lowering of the $\mathrm{w} / \mathrm{c}$ ratio (from 0.53 to 0.36 ), the use of polycarboxylate based superplasticizer, and the use of a calcium nitrate based accelerator. Figure 5 shows the compressive strength development of HES-ECC that more than meets the compressive strength targets. Over $25 \mathrm{MPa}$ was reached at about $4 \mathrm{~h}$ in HES-ECC. This strength is normally reached at about 3 days in ECC M45 (Wang 2005).

While the binder modification leads to an ECC with high early strength, it also results in a rapid diminishing of tensile strain capacity as a function of age, as shown in Fig. 6. The $4 \%$ strain capacity at $4 \mathrm{~h}$ was reduced to about $1 \%$ after 3 days. This suggests that the binder modification for high early strength leads to time-dependent micromechanical parameters that negatively impact the long term ECC tensile ductility performance, by violating one or both multiple cracking criteria (1) and (5).

To investigate the underlying reason for the progressive loss of tensile ductility, a detailed investigation ( $\mathrm{Li} 2009$ ) was launched to determine the time-dependence of micromechanical parameters. This is a case where the multiple cracking criteria were satisfied at early age, but continuous changes in matrix and/or fiber/matrix interface micromechanical parameters as a result of continuous hydration of binder leads to a gradual reduction of the ratio of $J_{b}^{\prime} / J_{\text {tip }}$ and/ or $\sigma_{0} / \sigma_{c r}$. Measurement of $K_{m}$ (Fig. 7) shows a rapid rise to about $0.8 \mathrm{MPa} \sqrt{ } \mathrm{m}$ at 28 days, surpassing the $0.64 \mathrm{MPa}$ $\sqrt{ } \mathrm{m}$ for the 28 day $K_{m}$ value for ECC M45 (Yang 2007) in just about 2 days. This rise of $K_{m}$ increases both $J_{\text {tip }}$ and $\sigma_{c r}$, and reduces the $J_{b}^{\prime} / J_{\text {tip }}$ and $\sigma_{\mathrm{o}} / \sigma_{\text {cr }}$ ratios. $J_{b}^{\prime}$ was shown to also decrease as a result of increased amount of fiber rupture due to time-dependent increase in interfacial bonds. These

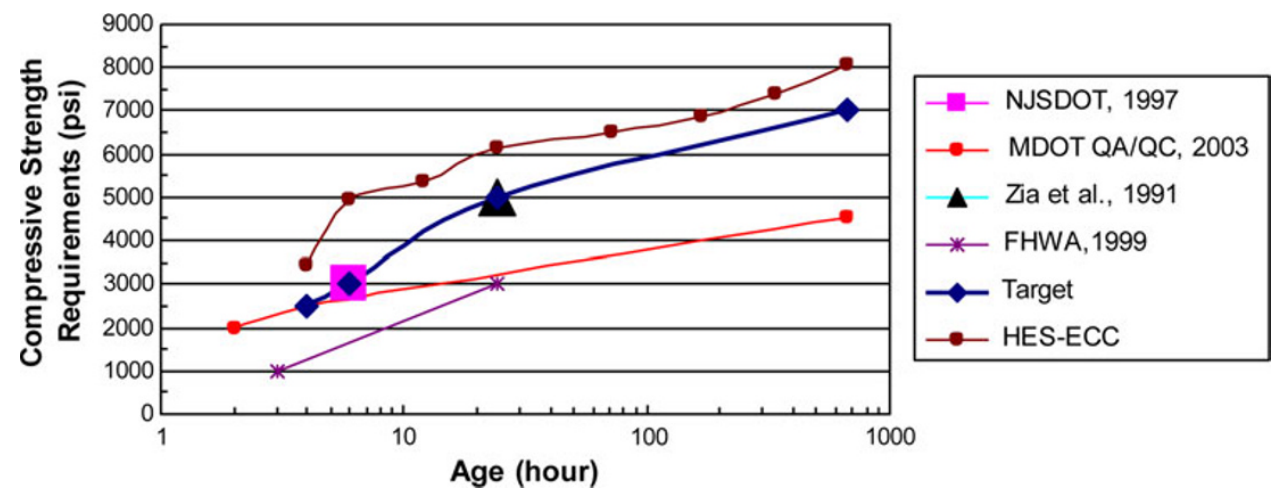

Fig. 5 Compressive strength development of HES-ECC. Also shown are requirements of high strength concrete specified DOTs and FHWA. 


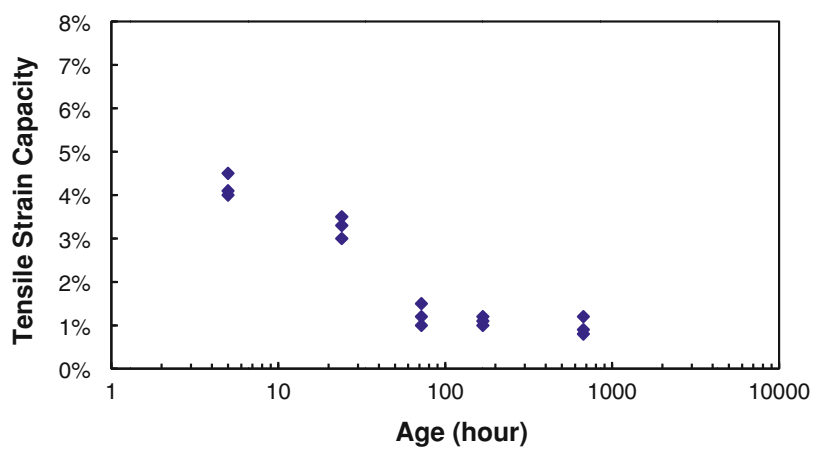

Fig. 6 Tensile strain capacity loss as a function of age prior to re-engineering of HES-ECC binder.

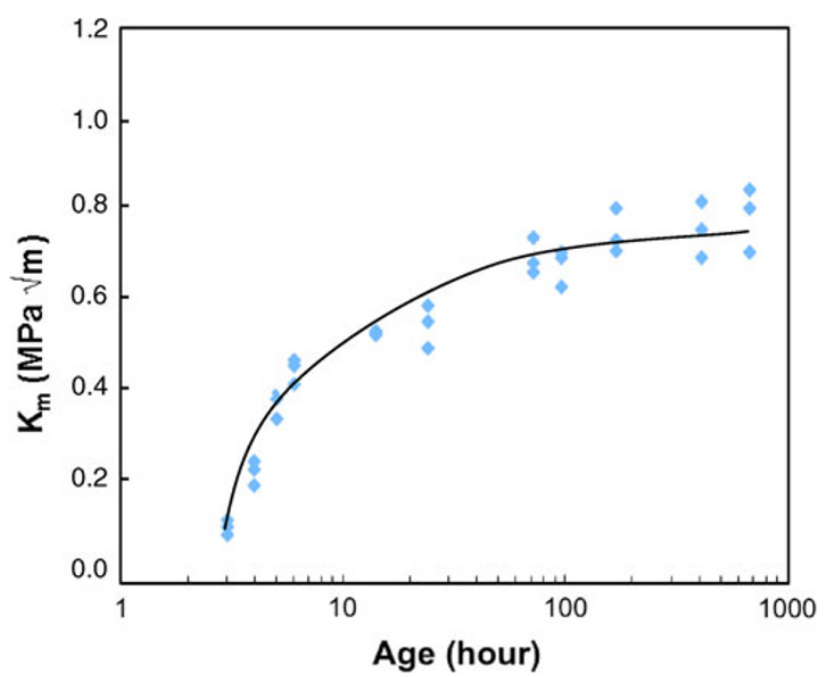

Fig. 7 Rise of $K_{m}$ as a function of age.

unintended changes in matrix and fiber/matrix interfacial properties are responsible for the loss of tensile ductility shown in Fig. 6.

Introduction of artificial flaws was investigated as a means to counteract the tendency of rising $K_{m}$. Polystyrene beads of $4 \mathrm{~mm}$ (Fig. 8) were chosen as artificial flaws that could trigger the initiation of multiple cracks more readily than relying on the intrinsic natural flaws. Still larger size would

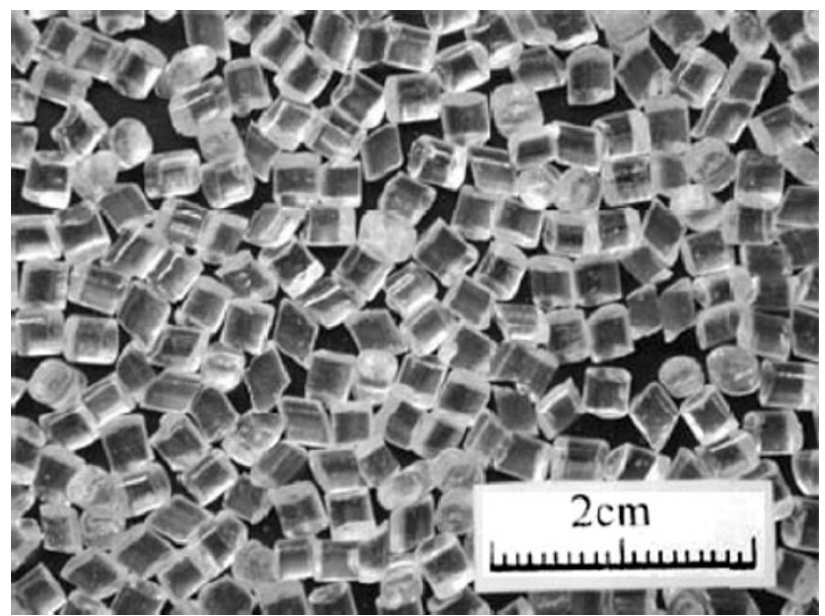

Fig. $84 \mathrm{~mm}$ polystyrene (PS) beads adopted as artificial flaws to restore tensile ductility in HES-ECC.

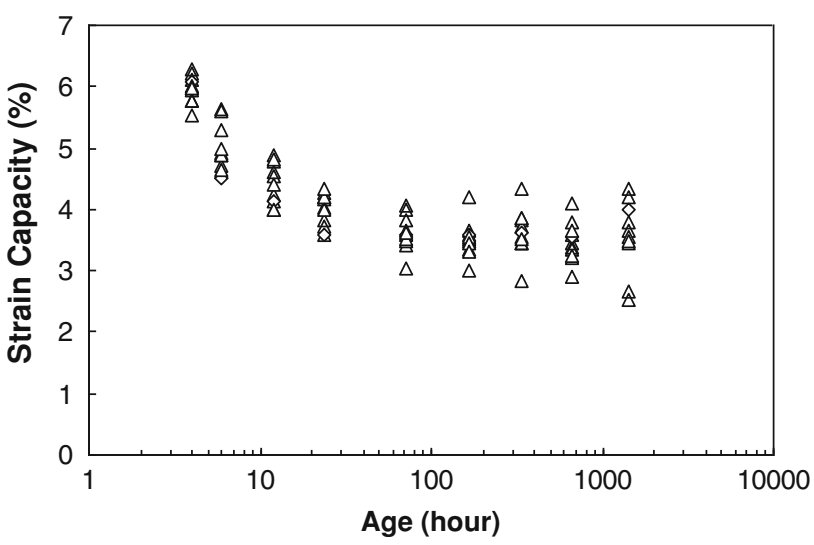

Fig. 9 Tensile strain capacity stabilized at about $3 \%$ after re-engineering of HES-ECC binder with artificial flaws.

have depressed the compressive strength. The result of the introduction of artificial flaws at $6.4 \%$ by weight of cement is summarized in Fig. 9, which shows a plateauing of tensile strain capacity at about $3 \%$ after three days. Figure 10 shows the tensile stress-strain curve and the saturated multiple cracking pattern of the HES-ECC specimen at 28 days. In HES-ECC, the introduction of artificial flaws of a size larger than the natural flaws successfully restores the tensile ductility.

\subsection{Self-healing ECC}

One of the well-known facts in concrete autogenous healing is the critical role played by the crack width. For ECC material, Li and Yang (2007) demonstrated negligible recovery of resonant frequency (RF) when the crack width is larger than $150 \mu \mathrm{m}$, partial recovery when the crack width is between 50 and $150 \mu \mathrm{m}$, and full recovery (100\% of RF of virgin specimen exposed to the same wet/dry cycles) when the crack width is below $50 \mu \mathrm{m}$. Although the healing performance would depend on the exact composition of the ECC and the exposure environment, this study shows that

(a)

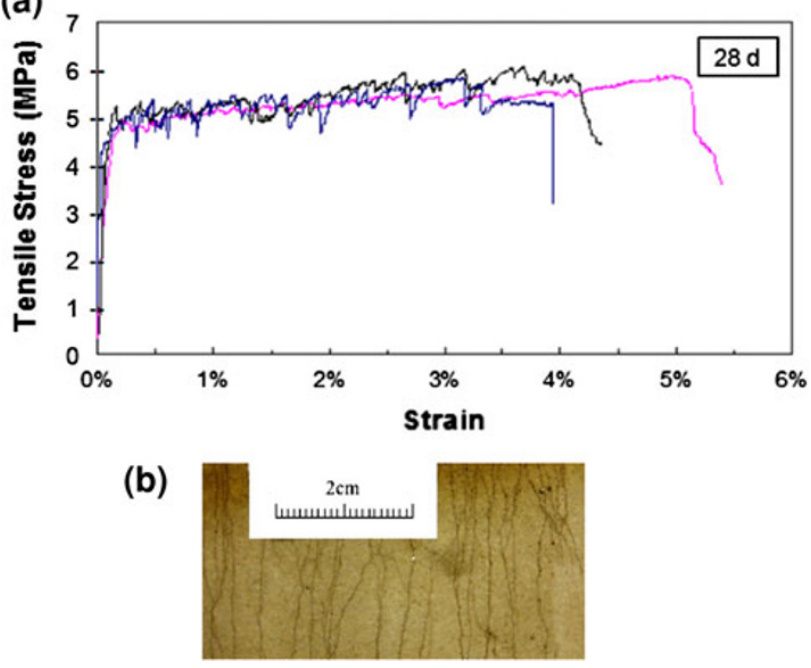

Fig. 10 a Stress-strain curve and b Crack pattern of HESECC at 28 days. 


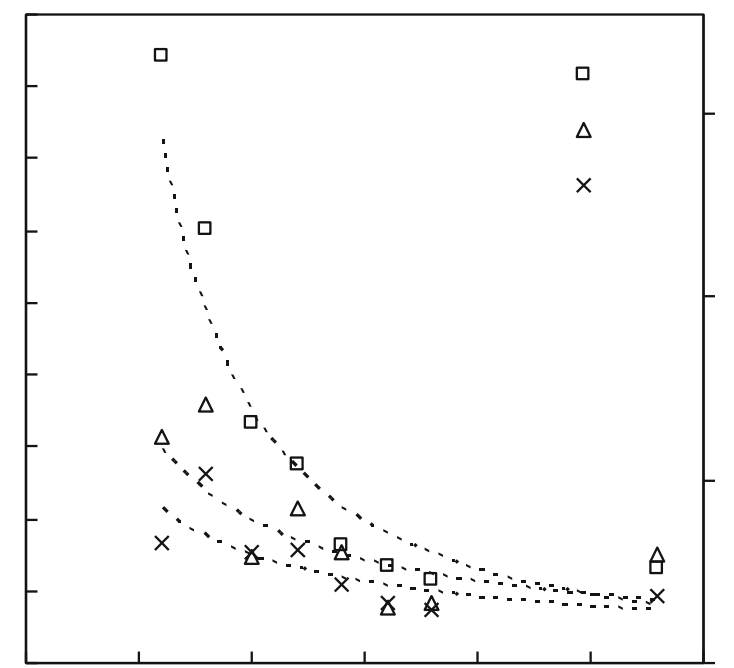

Fig. 11 Residual crack width of HFA ECC as a function of fly ash content at different ages.

ECC designed to have crack width below $50 \mu \mathrm{m}$ would be preferred for more robust self-healing.

One approach for designing ECC with ultra-tight crack width is to control the fiber/matrix interface bond. Figure 11 (Yang et al. 2007) shows reduction of the residual (after specimen unloading) crack width by increasing the FA/C ratio. Also shown in this plot is the dependency of the crack width on specimen age. At early age (3 days, for example) the crack width is higher due to underdeveloped fiber/matrix interface bond. Figure 12 shows the microcrack width for ECC with different FA/C ratios at the age of 3 days. For early age cracks, therefore, a $\mathrm{FA} / \mathrm{C}$ ratio above 2 would restrict the crack width to less than $50 \mu \mathrm{m}$ and allow more complete healing.
The underlying reason for the observed reduction in crack width with increase in $\mathrm{FA} / \mathrm{C}$ ratio is the reduction in fiber/ matrix chemical bond $G_{d}$ accompanied by an increase in frictional bond $\tau_{o}$ when higher FA content is introduced (Figs. 13a, b, Yang et al. 2007). There is a tendency for the round FA particles to agglomerate around the fiber surface. These interface bond modifications lead to a higher $J^{\prime}{ }_{b}$. Further, a lower $K_{m}$ is attained with increase in FA (Fig. 13c, Yang 2007). Both trends enhance the satisfaction of the multiple cracking criteria (1) and (5). Most importantly for self-healing, the higher fiber/matrix interface frictional bond leads to a tighter crack width. Figure 14 shows the computed $\sigma(\delta)$ relation for two ECCs at 28 days age. ECC M45 is predicted to have crack width varying between 27 and $95 \mu \mathrm{m}$. A high volume fly ash ECC (HFA-ECC) with FA/ $\mathrm{C}=2.8$ has computed crack width varying between 16 and $55 \mu \mathrm{m}$, using interfacial parametric values measured by Yang et al. (2007). These crack width ranges will be reduced by about $30-50 \%$ when residual values on unloading are considered. In these calculations, the crack width variation is derived from the assumption of fiber dispersion non-uniformity $(1.5-2.5 \%)$ in the uniaxial tension specimen. Sample images of residual crack pattern of these two ECCs are shown in Fig. 14.

Figure 15 shows the RF recovery of the self-healed HFA-ECC, compared to that of ECC M45 (FA/C = 1.2). The specimens were preloaded in tension to a severe $2 \%$ strain at 5 different ages (3, 7, 28, 56, and 90 days). After 4-5 cycles of wet/dry exposure, over $85 \%$ recovery of RF was observed in the HFA-ECC at all ages. For ECC M45 this number is about $77 \%$. Consistent with Fig. 11, which shows the largest difference in crack width for ECC with different FA content when damaged at younger age, the RF
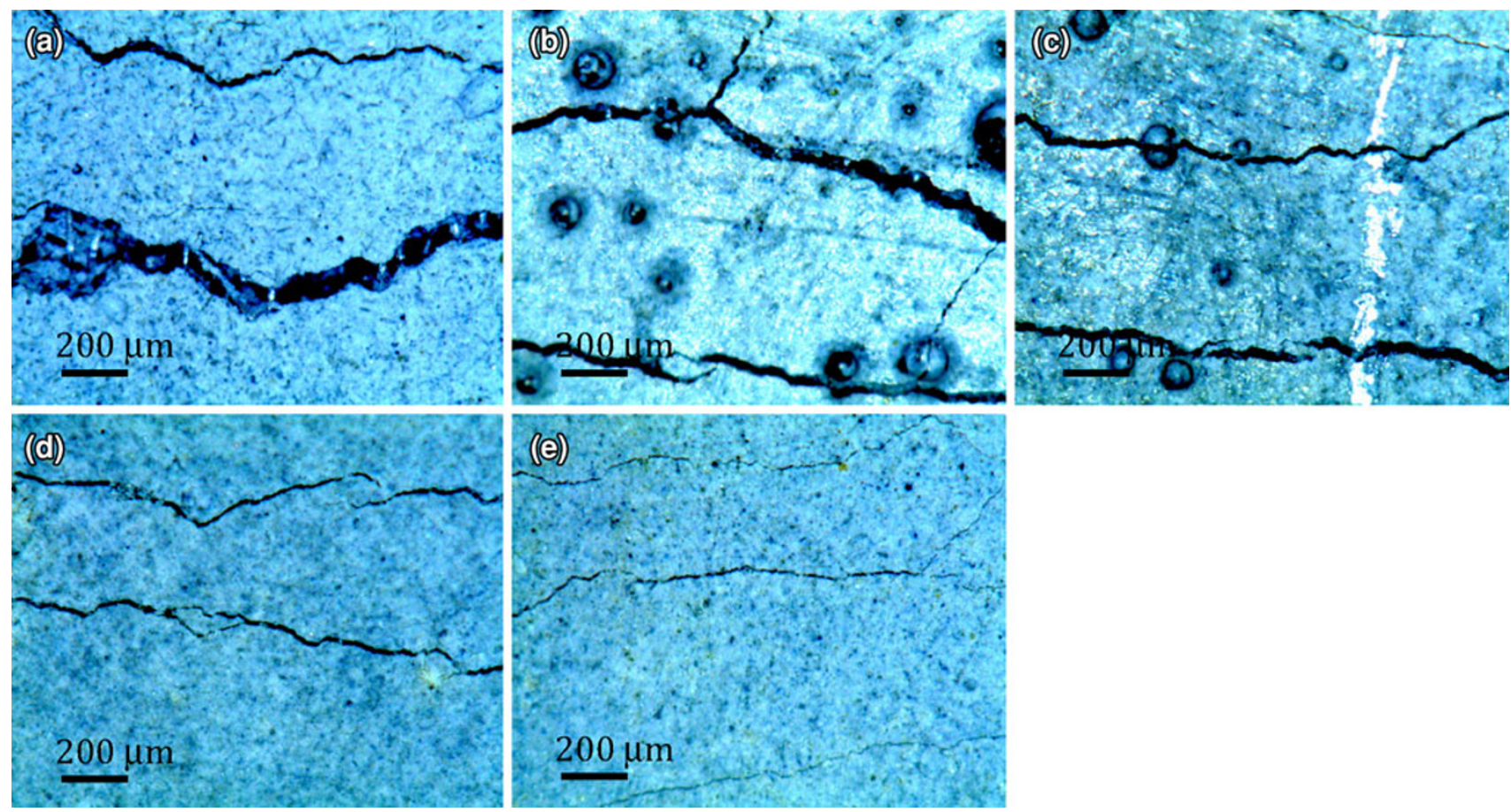

Fig. 12 Microscopic photos of residual crack width of HFA ECC at the age of 3 days where FA/C are a 1.2, b 1.6, c 2.0, d 2.8, and e 3.6, respectively. 

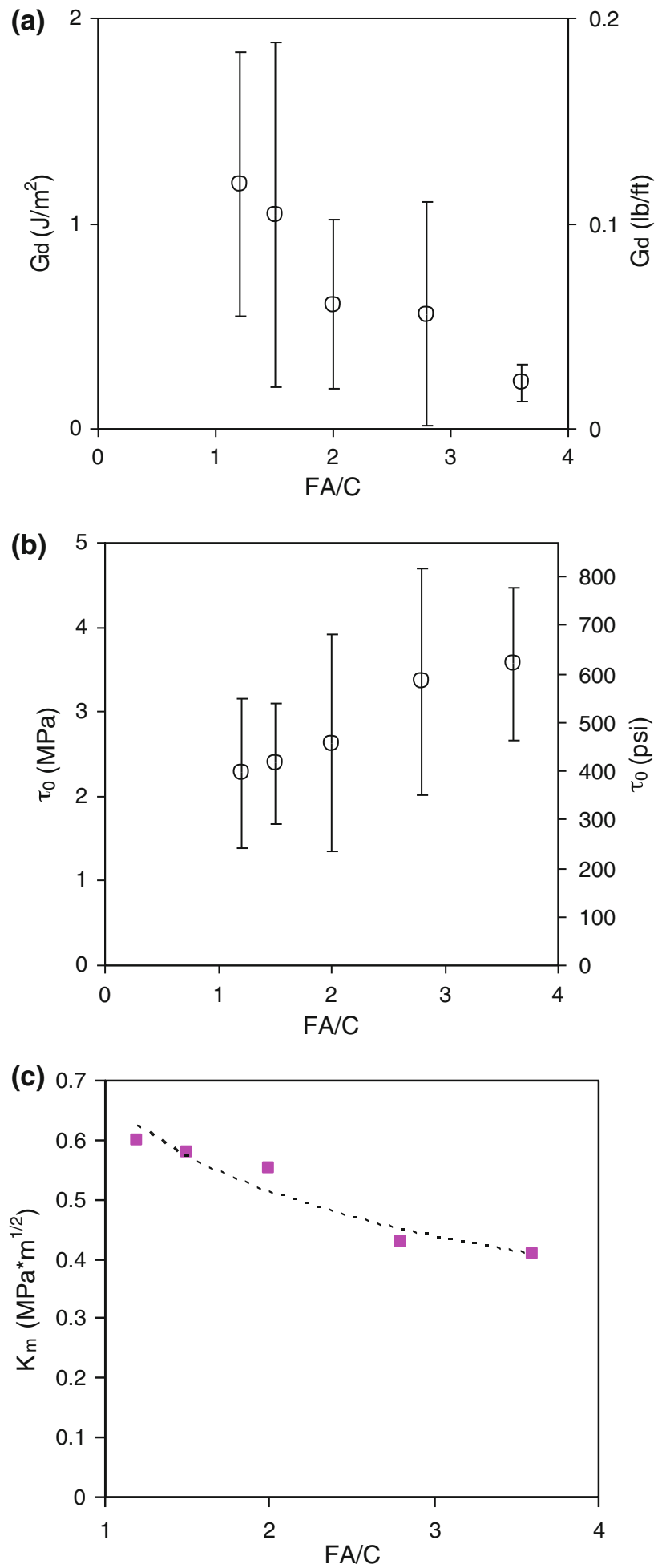

Fig. 13 a Chemical bond strength, $b$ frictional bond strength and $\mathbf{c}$ matrix fracture toughness of HFA ECC as a function of fly ash content at the age of 28 days.

recovery also tends to show a larger difference between ECC M45 and HFA-ECC at younger ages in Fig. 15. At lower preloading strain, e.g. at $0.3 \%$, both materials experienced over $95 \%$ RF recovery. Figure 16 shows the tensile stressstrain curves of the HFA-ECC under preloading and reloading after healing. It shows that the tensile strain capacity after healing remains over $3 \%$. For ECC M45 this number is about $2 \%$.

\section{Further Discussions and Conclusions}

In this article, the tailoring of ECC with various attributes or functions is reviewed. In general, the attainment of a certain desirable attribute/function may or may not impact the tensile ductility. If the tensile ductility is negatively affected, it is usually feasible to trace the fundamental source of the loss of ductility to specific fiber, matrix, or fiber/ matrix interface micromechanical parameters. Often times, modifications made to the matrix may also affect the fiber/ matrix interface, and vice versa. Micromechanics then provides guidelines for corrective actions. In this manner, the development of ECC with special attributes or functions can be attained in a rational and systematic manner, as opposed to by trial-and-error.

Three example ECCs are used to illustrate the micromechanics guided design. These are lightweight ECC, high early strength ECC, and self-healing ECC. In the case of lightweight ECC with density ranging from 0.93 to $1.45 \mathrm{~g} / \mathrm{cm}^{3}$, modification of the matrix density from that of ECC M45 was attained by the inclusion of low-density hollow glass bubbles. In the case of robust self-healing ECC, modification of the fiber/matrix interface from that of ECC M45 was attained by the inclusion of high volume of fly ash that tended to preferentially agglomerate around the fiber/ matrix interface and thus lowered the chemical bond $G_{d}$ while increasing the frictional bond $\tau_{o}$. The result is a stiffening of the microcrack bridging by fibers and a lowering of the crack width. The tighter crack width ECC then leads to more robust self-healing. In both cases of lightweight ECC and self-healing ECC, the modifications made to the composition of ECC M45 lead to a lowering of the matrix fracture toughness $K_{m}$ that is favorable to meeting the multiple cracking criteria set forth in Eqs. (1) and (5). In the case of high early strength ECC, the various modifications made to the matrix, including the replacement of Type I by Type III cement, result in a HES-ECC that attains over $25 \mathrm{MPa}$ compressive strength in $4 \mathrm{~h}$. However, the modifications also become detrimental to the time-dependent tensile ductility, reducing the tensile strain capacity from $3 \%$ to less than $1 \%$ over time. Micromechanical analyses trace this negative impact to the increase of $K_{m}$ over time, in addition to alterations in the fiber/matrix interface properties that reduce the $J^{\prime}{ }_{b}$ value over time. These changes in micromechanical parameters result in the violation of both multiple cracking criteria. The corrective action of artificial flaws introduction proved successful in restoring the long-term tensile ductility of HES-ECC to about $3 \%$.

The following specific conclusions can be drawn:

1. It is feasible to design special attributes or functionalities into ECC. In addition to the three discussed in this article, a variety of ECCs with other special attributes has been successfully designed.

2. When special attributes or functions are designed into ECC, the delicate balance between fiber, matrix, and interface engineered into ECC may be disturbed. Retailoring of ECC composition may be needed to 

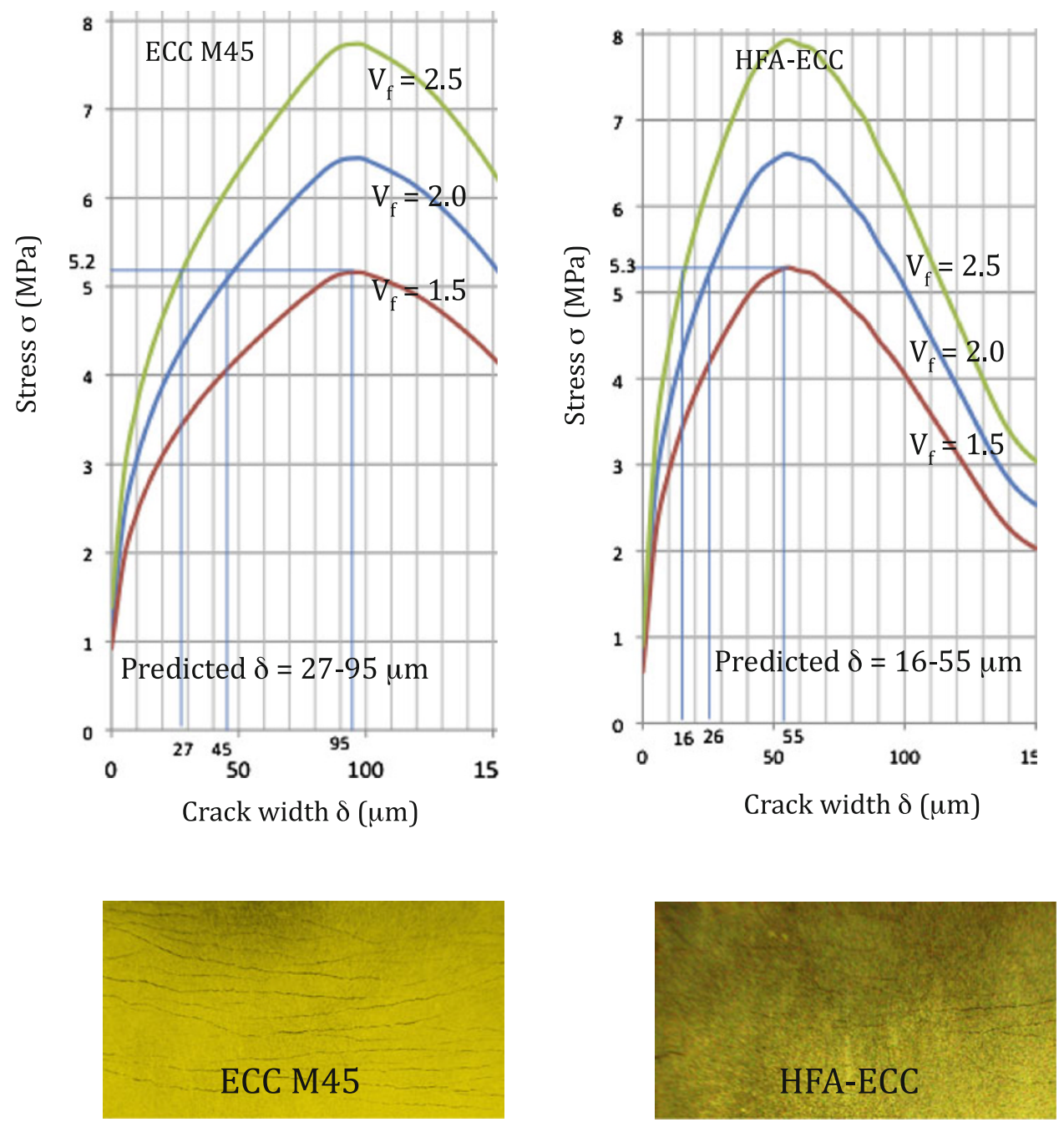

Fig. 14 Computed $\sigma(\delta)$ and crack width variation.

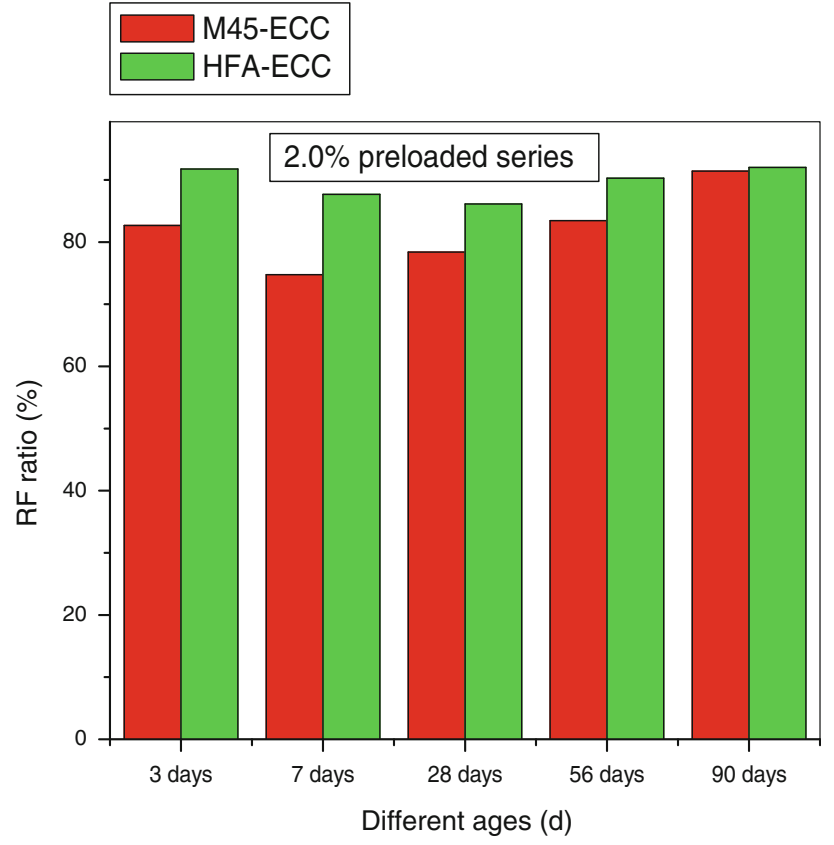

Fig. 15 Measured RF ratios (RF after damage and rehealing/ RF before damage) of ECCs preloaded to $2 \%$ strain at different ages. The tighter crack width HFA-ECC possess stronger rehealing ability, particularly when damage at early age.

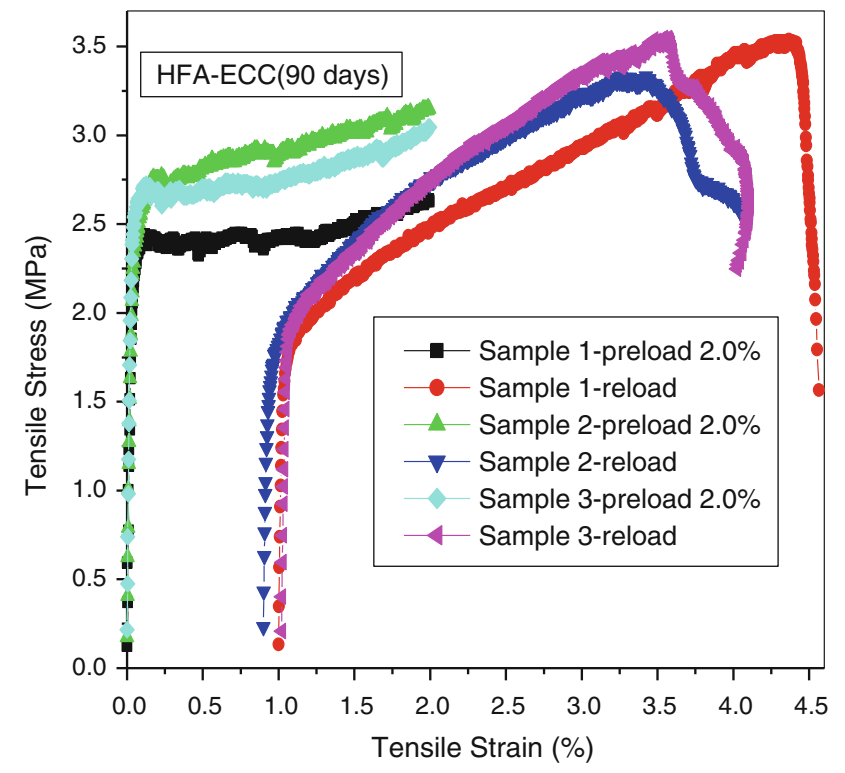

Fig. 16 Preloading and reloading curves of HFA-ECC damaged at 90 days age.

restore the unique high tensile ductility of ECC. Retailoring may target adjustments in fiber, matrix, and/or fiber/matrix interface. 
3. Micromechanics serves as a rational methodology for developing cementitious composites with high tensile ductility combined with special attributes or functions. Micromechanics provides a tool to systematically trace the source of ductility loss, if any, and offers guidelines to adjust compositions to restore composite ductility.

\section{Acknowledgments}

Partial support for this work has been received from the US National Science Foundation (CMMI 1030159, 1068005), The National Institute of Science and Technology-Technology Innovation Program (NIST-TIP Cooperative Agreement: 70NANB9H9008), and from the University of Michigan.

\section{Open Access}

This article is distributed under the terms of the Creative Commons Attribution License which permits any use, distribution, and reproduction in any medium, provided the original author(s) and the source are credited.

\section{References}

Horikoshi, T., Ogawa, A., Saito, T., \& Hoshiro, H. (2006). Properties of polyvinylalcohol fiber as reinforcing materails for cementitious composites. In Proceedings of International RILEM Workshop HPFRCC in Structural Applications (pp. 145-153). Paris: RILEM SARL.

Kanda, T., \& Li, V. C. (2006). Practical design criteria for saturated pseudo strain hardening behavior in ECC. Journal of Advanced Concrete Technology, 4(1), 59-72.
Li, M. (2009). Durability of concrete repair through ductility and crack width control in ECC. PhD thesis, the University of Michigan.

Li, M., \& Li, V. C. (2011). High-early-strength ECC for rapid durable repair: Material properties. ACI Materials Journal, 108(1), 3-12.

Li, V. C., Wang, S., \& Wu, C. (2001). Tensile strain-hardening behavior of PVA-ECC. ACI Materials Journal, 98(6), 483-492.

Li, V. C., Wu, C., Wang, S., Ogawa, A., \& Saito, T. (2002). Interface tailoring for strain-hardening PVA-ECC. ACI Materials Journal, 99(5), 463-472.

Li, V. C., \& Yang, E. H. (2007). Self-healing in concrete materials. In S. van der Zwaag (Ed.), Self healing materials: an alternative approach to 20 centuries of materials science (pp. 161-193). Heidelberg: Springer.

Lin, Z., \& Li, V. C. (1997). Crack bridging in fiber reinforced cementitious composites with slip-hardening interfaces. Journal of Mechanics and Physics of Solids, 45(5), 763-787.

Wang, S. (2005). Micromechanics based ECC matrix design. $\mathrm{PhD}$ thesis, the University of Michigan.

Wang, S., \& Li, V. C. (2003). Materials design of lightweight PVA-ECC. In A. E. Naaman \& H. W. Reinhardt (Eds.), Proceedings of HPFRCC (pp. 379-390), Ann Arbor.

Wu, H. C., \& Li, V. C. (1999). Fiber/cement interface tailoring with plasma treatment. Journal of Cement and Concrete Composites, 21, 205-212.

Yang, E. H. (2007). Designing functionalities into ECC materials via micromechanics, $\mathrm{PhD}$ thesis, the University of Michigan.

Yang, E. H., Yang, Y., \& Li, V. C. (2007). Use of high volumes of fly ash to improve ECC mechanical properties and material greenness. ACI Materials Journal, 104(6), 303-311. 